\title{
Voyageur écrivain, voyageur romanesque: Un phénomène de littérature de voyage
}

\section{Traveler Writer, Romanesque Traveler: A Travel Literature Phenomenon}

\author{
Abdulfettah IMAMOĞLU' (B)
}

'Dr., Eskişehir Osmangazi University, Faculty of Arts, Department of Comparative Literature, Eskisehir, Turkey

ORCID: A.I. 0000-0003-3509-066X

\section{Corresponding author:}

Abdulfettah İMAMOĞLU,

Eskişehir Osmangazi Üniversitesi, Fen Edebiyat Fakültesi, Karşılaştırmalı Edebiyat Bölümü, Eskişehir, Türkiye

E-mail: aimamoglu@ogu.edu.tr

Submitted: 04.07 .2020

Revision Requested: 31.08 .2020

Last Revision Received: 07.09.2020

Accepted: 29.09 .2020

Citation: Imamoglu, A. (2020). Voyageur écrivain, voyageur romanesque: Un phénomène de littérature de voyage. Litera, 30(2), 489-503.

https://doi.org/10.26650/LITERA2020-0096

\begin{abstract}
RÉSUMÉ
L'adjectif "romanesque" qui vient du mot "roman" et qui signifie, dans un sens élargi, quelque chose sortant de l'ordinaire, ou quelque chose relevant du rêve et de l'imaginaire peut être pris comme un terme principal pour comprendre la situation du voyageur écrivain qui quitte son chez-soi dans l'intention de découvrir ce qui se situe au-delà des limites de sa sphère habituelle. Le récit de voyage offre, en ce sens, une perspective considérable relativement à la perception de la réalité quand il s'agit d'acquérir une expérience dans un espace inconnu avec des conditions imprévisibles. Dans un tel genre de récit, l'espace et la personnalité font l'objet d'une relation exceptionnelle et éventuelle qui se développe sous une condition d'étrangeté étant liée aux circonstances de l'ailleurs d'une part, et à l'état individuellement changeant du voyageur d'autre part. Ainsi, un habitant ordinaire devient, d'abord, voyageur après avoir quitté son chez-soi, puis, devient voyageur écrivain en conséquence de son acte de rapporter son expérience physique, et finalement, voyageur romanesque, lorsque cette expérience de voyage se traduit en des mots qui font apparaitre un nouveau monde dans lequel le voyageur écrivain prend le rôle d'un observateur attentif qui voit dans les choses ce qui n'est pas auparavant vu, ce qui est caché, ce qui porte les traits de l'imaginaire et du sensationnel. En se référant aux ouvrages littéraires comportant le thème de voyage, réalisés au cours des deux derniers siècles, le but sera ici de mettre en évidence, en tant que phénomène de littérature de voyage, la possibilité d'une association conceptuelle et d'une relation conséquente par rapport aux termes "voyageur écrivain" et "voyageur romanesque".
\end{abstract}

Mots-clés: Littérature de voyage, littérature comparée, littérature du vingtième siècle, littérature du vingt-et-unième siècle, poétique de l'espace

\section{ABSTRACT}

The French adjective "romanesque", which derives from the French word for "novel" and which means, in a broader sense, something out of the ordinary or something that derives from dream and imagination can be taken as a principal term to understand the situation of the traveler writer who leaves her/his home to discover beyond the limits of her/his habitual sphere. Travel writing offers, at that point, a notable prospect regarding the reception of the reality when it comes to have an experience in an unknown place with unpredictable conditions. In this sort of 
writing, the space and the personality take part in an eventual and exclusive relation under the condition of strangeness related to the circumstances of elsewhere and to the changing individual state of the traveler. Thus, an ordinary habitant becomes, first, traveler when $\mathrm{s} / \mathrm{he}$ leaves the home, then, becomes traveler writer when $\mathrm{s} / \mathrm{h}$ e reports her/his experience, finally, "romanesque traveler" when her/his psychical travel experience gives a wordily result through creating a new world in which the traveler writer takes the role of attentive observer who tends to see in things the unseen, the hidden, the imaginative and the sensational. By referring to the literary works, with the theme "travel", that have been realized throughout the twentieth and the twenty-first centuries, this paper aims to show a possible conceptual association and a consecutive relation that occur between the titles "traveler writer" and "romanesque traveler" as a phenomenon of travel literature.

Keywords: Travel literature, comparative literature, the twentieth century literature, the twenty-first century literature, poetics of space

\section{EXTENDED ABSTRACT}

The act of traveling, which, in the context of this work, can be defined as an act of roaming or wandering as well, indicates a process which, from the beginning, is defined by a sort of state of imprecision. After the words of traveler writers, a decision to leave the home, that space offering established norms and limits, seems to involve a shortage of precision in terms of destination, the name which designates the place to arrive by providing, at the same time, a semblance of limited situation. Following that decision with no definitive arrival point, the traveler writer becomes part of a different spatial condition in which her or his senses pass into a new phase. In the first chapter entitled "Going elsewhere: an aleatory decision", this uncertain origin of the decision to go elsewhere will be correlated to a romanesque quality as it makes the travel an unpredictable, emotional and temporary event. After that, in the second chapter called "Romanesque manner of being in space", a consideration will be delivered about the behavior of the traveler writer in relation with its exceptional changing because of the new manifestation of elements of the world. That being so, elsewhere will be taken according to its provocative side that generates a kind of metamorphosis which takes place under the unfamiliar circumstances. Correspondingly, it will be shown that the traveler writer represents an unpremeditated and extraneous presence as $s / h e$ has the impression to move, during her or his stay in elsewhere, without a solid apprehension of orientation. Given the term romanesque manner of being in space is used here, especially, as a term to denote, by referring to a language that is designed to translate the eccentricity of the moments that have inevitably marked an erratic presence of a perceiver subject, the relatively isolated being of the traveler writer before an unwonted and stimulating authenticity of space. Therefore, as a literary product, travel writing is ascribed to contain fragments that reveal those particular moments lived by the traveler 
writer in an unusual way and it consequently becomes the final and the most concrete prove of such a metamorphosis that describes a different state of being in space, an alterity that underscores the value of a "sincere experience" which gives way to a "confrontation of the real and the imaginary". Through this process of transmission and transformation of the experience into words and wordily descriptions, the traveler writer becomes a romanesque traveler as s/he reports to the lecturer the wondering character of her or his individually realized act and travel writing becomes the extraordinary history of this Other who appears to discover the dissimilar and diverse reality of elsewhere. Altogether, through the words of traveler writers representing the two last centuries and different countries and languages, be they French (Canada, France, Swiss and Togo) and Turkish, the act of traveling, in this work, will be reinterpreted after what is suggested in travel writing that presents to the reader an unorthodox figure who is attracted by strangeness, with her or his loose motivation to move further, and who finds herself or himself in a position to see what stands outside the wellregulated path of habitual life. According to what is depicted and formulated about that distinctive state of being in space, which is defined, from the beginning, by imprecise traits and evolved, afterwards, through an unforeseeable set of events, in this paper, the main argument will be that the title "romanesque traveler" takes shape of a term to be the most convenient appellation to make understandable the change of traveler writer that takes place in relation with a surprisingly transformed spatial reality. 


\section{Introduction}

Il est possible, en des termes les plus simples, de définir "voyageur écrivain" comme une personne qui voyage, qui prend des notes lors de son voyage tout en acquérant des souvenirs, et qui transforme ces notes/souvenirs en une œuvre littéraire afin de transmettre son expérience personnelle, à travers les mots, au lecteur qui n'a pas été avec lui lors de cette expérience mais qui obtient la chance de resuivre, d'après une transformation du voyage en mots de récit, l'itinéraire réalisé de l'écrivain. Lorsque le voyage se transforme en mots et devient ainsi une œuvre écrite, un monde écrit attendant, de la part du lecteur, d'être découvert, le voyageur devient alors écrivain. Autrement dit, le récit de voyage affirme les titres "voyageur" et "écrivain" de celui qui relate sa propre expérience de voyage d'après ses propres mots.

Le voyageur écrivain est celui qui peut être capable de rendre l'acte de voyage littéraire et romanesque. II peut le rendre romanesque parce que le voyage individuel de l'écrivain obtient, dans son ouvrage, un aspect non seulement et simplement visible mais aussi des aspects psychique, corporel, révélateur, imaginaire, extraordinaire, etc. Ces aspects deviennent, bien entendu, manifestes grâce à l'expression de l'écrivain qui offre au lecteur une version romanesque de son voyage réalisé.

Outre son sens lié strictement et formellement au genre de roman, le mot "romanesque" est défini dans les dictionnaires comme le suivant:"qui évoque le roman par ses aventures extraordinaires, ses péripéties nombreuses, ses rebondissements imprévus, sa destinée exceptionnelle". Une deuxième acception s'appuie sur l'aspect encore plus général du terme: "ce qui sort de l'ordinaire", ou encore "qui relève du rêve". En tenant compte de ces deux dernières définitions proposées pour le mot "romanesque", puisque ce sont ces deux explications lexicales qui nous intéressent au juste, cet article vise à démontrer le caractère romanesque des récits de voyage relativement aux particularités discursives et rhétoriques que l'on trouve dans ces sortes d'écritures littéraires qui ne sont pas classifiées en tant que romans, qui portent pourtant des traits romanesques.

Pour traiter le sujet de ce travail, ont été choisis des récits de voyage et des récits traitant le thème de voyage, écrits au cours des vingtième et vingt-et-unième siècles, afin de présenter d'une perspective moderne et contemporaine un profil de voyageur écrivain qui pourrait être plus pertinent par rapport au temps actuel où le voyage est 
perçu, au-delà d'être une mission ou une aventure, comme une expérience révélatrice en ce qui concerne la personnalité corporelle, psychique et conceptuelle de l'individu humain à titre de voyageur. Dans le choix de ces récits, on propose également une diversité temporelle et culturelle, représentant les littératures française, canadiennes, togolaise, suisse et turque, dans l'intention de représenter un plus grand nombre de différences et d'individualités.

Dans cet article, le voyage de l'écrivain sera envisagé par rapport à son caractère qui sort de l'ordinaire, de l'habituel et du prévu. Ainsi, dans le premier chapitre intitulé "Partir ailleurs: une décision aléatoire", I'accent sera mis sur la motivation de départ du voyageur écrivain qui témoigne d'un état d'imprécision. Ensuite, dans le deuxième et dernier chapitre nommé "Une manière romanesque d'être dans l'espace", la notion romanesque sera considérée par rapport à la conception spatiale du voyageur écrivain qui exprime ce qu'il a vécu pendant le moment où il se trouvait dans l'autre lieu. Ensemble, sous sa forme littéraire, le voyage sera examiné à partir de différents stades par lesquels l'expérience de déplacement du voyageur écrivain acquiert un aspect romanesque.

\section{Partir ailleurs: une décision aléatoire}

Quand il s'agit de voyager, celui qui voyage peut avoir des noms divers destinées à définir la nature de son acte de déplacement. Or, cet acte peut varier selon l'itinéraire, le but, le comportement, le point de vue et la vision du voyageur. Ce dernier est ainsi appelé "vagabond" et son acte comme "vagabondage", ou encore "errance" ou "déambulation", un ensemble terminologique pour insister sur le côté incertain et spontané des décisions prises par l'écrivain voyageant dans l'ailleurs sans but vraiment précis. "Sait-on jamais le véritable motif d'un départ pour un grand voyage?", se poset-il la question, à ce sujet, le voyageur écrivain togolais, Tété-Michel Kpomassie qui assume que "tant de causes, de mobiles ou d'impulsions s'enchevêtrent pour donner lieu à un semblant de raison" (1981, p. 306).

Fournir une explication à la décision du voyageur qui quitte son chez-soi pour partir ailleurs n'est sans doute pas une entreprise simple à effectuer. Car cette décision révèle, dès le début, un état imprécis. Le moment de montrer la volonté pour réaliser une telle action de déplacement, décider l'itinéraire, déterminer un schéma de temps désignent, tous, les différents stades où la condition indéterminée du voyageur écrivain devient 
d'autant plus sensible que ce dernier devient ouvert à des circonstances aléatoires par lesquelles son acte de voyage prend une forme dépourvue de lignes bien définies. Ainsi, l'endroit que l'on peut appeler "destination", désignant sans doute un lieu avec des limites plus ou moins précises pourvue que le voyage de l'écrivain doit avoir lieu dans les limites du monde (en l'occurrence) qui comprend des territoires formés à partir des frontières à caractère déterminé, devient équivalent d'un semblant de point d'arrivée en raison du fait que ce pseudo-point d'arrivée, si l'on peut le nommer ainsi, ne possède guère, selon les mots du voyageur écrivain, ce que doit être qualifié comme un point d'arrivée, un endroit où le voyageur peut ressentir un sentiment d'achèvement définitif.

La précision, qui peut être associé à l'adjectif"ordinaire"en raison de son lien suggestif avec le mot "ordre", n'a pas de véritable correspondance dans la terminologie personnelle du voyageur écrivain qui se déplace d'une manière aléatoire. Même si les limites, les territoires et le monde correspondent à des espaces qui indiquent relativement une précision et qui ont d'ailleurs besoin des précisions, l'espace dans lequel se déroule le déplacement du voyageur écrivain semble être dépourvu d'un sens bien déterminé de la précision malgré les limites qui le détermine dans un cadre géopolitique. Puisqu'elle donne au voyage un caractère imprévisible, cette condition d'imprécision peut se traduire en tant que romanesque. Or, quand Sylvain Tesson affirme que le chemin du voyageur est "pavé d'imprévu" (2017, p. 54), et lorsque, plus tôt, un autre voyageur écrivain, Nicolas Bouvier dit que "la vérité, c'est qu'on ne sait comment nommer ce qui vous pousse"; "quelque chose en vous grandit et détache les amarres, jusqu'au jour où, pas trop sûr de soi, on s'en va pour de bon" (2001, p. 12), on se procure au juste de matériel romanesque qui indique clairement un processus d'action qui sort de l'ordinaire, qui manque de précision. C'est la raison pour laquelle, semblable au cas du point d'arrivée, l'action initiale du voyage, qui définit donc le départ, comporte, elle aussi, des traits pas très nets. Ici, il s'avère que le voyageur a mal à formuler une explication satisfaisante, que ce soit pour le lecteur ou pour soi-même, au contraire, il accentue l'aspect confus de son action. Encore, de plus de ces voyageurs contemporains qui évoquent de façon suffisamment compréhensible l'aspect individuellement incertain du voyage, le voyageur écrivain turc Enis Batur, pour aborder ce même sujet, mais cette fois à partir d'une formulation poétique, s'exprime en ces termes: "derrière moi s'étend la route que j'ai faite, devant moi se montre un nuage de poussière" (2011, p. 154). Toutes ces affirmations rendent difficiles, par conséquent, à saisir les traits d'une probable description du début du voyage. 
Le chemin du voyageur écrivain s'étend devant lui comme une métaphore de l'inconnu et de l'extraordinaire. La dualité conceptuelle de l'espace, soient "espace découvert" contre "espace à découvrir", indiquée ci-dessus par le terme "nuage de poussière" de Batur s'inscrit, à cet égard, dans une conception de l'espace d'après laquelle la notion d'ailleurs prend nécessairement un sens distinct mais aussi un sens romanesque en comparaison avec la notion de chez-soi qui signifie, elle, un espace de caractère établi. Ainsi, pour découvrir l'ailleurs, c'est-à-dire le non-chez-soi, le voyageur parcourt alors ce chemin qui le mène vers ce qui s'oppose à l'ordinaire, vers ce qui se trouve en dehors des limites du familier que représente le chez-soi. Ce chemin, qui offre au voyageur une autre réalité de l'espace, une réalité extraordinaire, se figure en quelque sorte comme l'image d'un autre monde dans lequel les normes établies, le comportement ordinaire, la vision traditionnelle n'ont pas de valeur habituelle, ils n'y trouvent pas de véritables correspondances. Dans un tel contexte, quand une Gabrielle Roy énonce que longtemps elle a voyagé "sans boussole" et en prononçant ensuite la question que "pour la traversée de la vie, que vaut une boussole?" (2012, p. 300), c'est forcément la question de l'intérêt de la certitude des mesures qui s'impose une fois de plus par rapport à la conception de l'espace. De là, il paraît que l'expérience spatiale de l'ailleurs procure la possibilité exceptionnelle de vivre dans l'espace sans boussole, sans rester contraint à suivre l'itinéraire très précis de la vie ordinaire.

L'ensemble des termes cités jusqu'ici fait partie d'un contexte qui n'est pas compatible avec un langage que l'on peut définir comme formel, ordinaire ou strictement réaliste. Il s'agit en ce cas d'un langage qui est plus proche à un état indécis, ouvert aux changements abrupts ou surprenants. L'état de voyage s'écarte ainsi de la réalité de la vie quotidienne qui a lieu dans le chez-soi du voyageur écrivain. Or, dans la réalité du chez-soi, l'espace désigne le prévu et le stable, il se réfère à un ensemble de circonstances qui déterminent une sphère dont les limites répondent aux attentes de celui qui l'habite. Afin de se trouver en dehors de sa sphère habituelle, le voyageur écrivain, en tant qu'habitant, prend donc la décision de partir ailleurs et cette décision aléatoire définit le déroulement de son déplacement qui, sans suivre une direction catégorique, est marqué de moments imprévisibles. En raison des circonstances qui se manifestent d'une manière indéterminable, il convient de dire qu'en voyage c'est une autre manière d'être dans l'espace qui a lieu, et cette autre manière peut être décrite sous l'adjectif "romanesque", si bien que le voyageur écrivain parle, dans son récit de voyage, d'une sorte de présence qui est dissemblable à sa propre présence ordinaire. 


\section{Une manière romanesque d'être dans l'espace}

Le voyageur écrivain perçoit ce qu'il entoure d'un regard qui n'est pas le même quand il se trouve chez lui. C'est un "regard neuf" sur le monde, c'est une différente perspective de concevoir l'espace. Dans l'ailleurs, les sens du voyageur sont plus que jamais ouverts à ce qui se manifeste dans l'espace. Or, lorsque les éléments de l'espace peuvent passer inaperçus dans la condition du chez-soi, dans la spatialité exceptionnelle de l'autre lieu, ce qui constitue l'espace peut provoquer fortement la perception du voyageur en tant sujet étranger étant capable de sentir la différence. À ce propos, justement, Kpomassie parle du "rôle d'observateur attentif" du voyageur qui "demande sans cesse une tension d'esprit", aussi bien qu'il parle de la situation du voyageur étant "différente de celle des habitants" qui sont plongés dans leur monde et dans leur "petit cercle de vie" (1981, p. 159). Le cercle dont parle l'écrivain togolais représente ici une manière établie d'être dans l'espace et en dehors de ce cercle est situé un monde "sans boussole" ou bien, un monde "poussiéreux" qui transforme l'habitant ordinaire en voyageur romanesque. II devient un voyageur romanesque parce que ce dernier se rend compte de la différence entre la vie en tant qu'habitant, c'est-à-dire une vie qui se répète continuellement dans un ordre établi et une vie qui n'est guère associable avec une définition comme produit d'un langage résolu.

En éloignant de l'espace qui garantit et renforce son statut habitant, le voyageur écrivain fait désormais partie d'un monde indéterminable qui, avec ses éléments physiques ou psychiques, provoque sans cesse les sens et la perception. "Le voyage constitue le terrain idéal de la nouveauté", explique Tesson, selon qui l'incertitude du sort du voyageur "l'oblige à se tenir en éveil", car "chaque pas peut cacher une chaussetrappe, chaque kilomètre et chaque minute recéler le germe de l'improbable". Le voyageur français décrit d'ailleurs le voyage comme"l'intervalle entre les habitudes de I'homme" (2017, p. 54), et cela, il n'y aurait pas de doute, pour faire référence à une distinction concernant l'état de voyage par opposition à la vie habituelle qui se déroule à l'intérieur des limites bien connues d'un espace certain. Une remarque de tel type atteste que ce qui rend distinct l'espace que l'on peut par nécessité appeler "non-chezsoi", c'est la rupture avec les habitudes, c'est l'inclination à l'improbable. Quand il se trouve dans la spatialité de ce non-chez-soi, le voyageur écrivain entre donc dans une différente mode de vie d'après laquelle sa conception de l'espace prend d'autres sens, elle devient diverse. 
Le voyage change le regard, évolue la vision, éveille les sens, témoignent-ils les voyageurs écrivains. Apparemment c'est une manière différente de vivre, une autre manière d'être présent dans l'espace. Quand la voyageuse écrivaine Blanche de Richemont exprime, à ce titre, l'idée que "les vagabonds ne se contentent pas de voir le monde, ils le pénètrent", elle veut sans doute préciser cette différence, ce changement en ce qui concerne la perception du monde. Selon Richemont, le regard des vagabonds "est fertile car ils perçoivent la double vie qui sommeille en chaque chose. Ils saisissent I'universel dans le moindre détail. Où qu'ils se trouvent, ils sont en voyage. Chaque lumière est une découverte, une prière ou un poème" (2015, p. 110). Par le ton abstrait de la description qui se rapporte au côté heuristique du voyage, ou du vagabondage, on dessine dans cette formulation le voyageur écrivain comme un personnage romanesque qui ne voit pas le monde comme on le verrait normalement.

Les mots "découverte" et "le regard fertile" qui viennent d'être cités participent, lorsqu'il s'agit du voyage, d'une dialectique qui traduit l'état particulier du voyageur écrivain pendant le moment où il se trouve dans la condition temporaire de l'ailleurs. En différence avec la condition du chez-soi, le temps qui se passe dans la spatialité de l'ailleurs représente un état de découverte, une occasion spéciale de découvrir l'espace en se confiant pleinement à ses sens, de voir le monde autrement. Quant au terme "double vie", en tant que découverte résultant d'un regard fertile, souligne, peut-être d'une manière trop métaphysique mais assez convenable, la diversité de la disposition perceptuelle que contient chaque élément spatial. Cette même double vie des choses fut donc découverte d'après les sens en éveil du vagabond qui s'empare du rôle d'observateur à titre exceptionnel pendant son expérience de voyage. Et c'est encore dans une telle situation exceptionnelle que "saisir l'universel dans le moindre détail", autrement dit saisir l'aspect entier de chaque détail qui se manifeste aux sens du voyageur devient réalisable. Or, ce côté "universel" exige un regard fertile, un regard qui sort de l'ordinaire et qui est enfin capable de voir le monde autrement.

Parallèlement à cette position qui proclame la particularité sensiblement dépaysante de l'ailleurs, Roy interprète de façon suivante l'une de ses expériences vécues en dehors du chez-soi: "cette sensation de dépaysement, de pénétrer, à deux pas seulement de chez nous, dans le lointain, m'était plutôt agréable, quand j'étais enfant. Je crois qu'elle m'ouvrait les yeux, stimulait mon imagination, m'entraîner à observer" (2012, p. 11). Le témoignage de Roy explique adroitement ce que peut signifier la manière d'être dans l'espace autrement, car cette expérience vécue à l'âge de l'enfance s'est passée non 
loin du chez-soi, néanmoins, elle a révélé un temps de voir le monde d'un regard neuf, d'un regard qui a laissé sa marque dans la conception de l'espace de l'écrivaine. Dans sa description, la voyageuse canadienne fait l'usage des mots « observation » et "imagination" par lesquels peut se comprendre la position du voyageur envers ce qui se manifeste d'une manière différente et attirante dans l'espace. Ici, le côté "stimulant" du déplacement, comme le précise Roy, peut, encore une fois, être associé au regard fertile du voyageur écrivain qui a ses sens éveillés conformément à une expérience de découverte inhabituelle. II s'agit là d'un nouvel ensemble d'éléments spatiaux qui évolue la perception du voyageur écrivain et conduit éventuellement sa présence à un rôle extraordinaire par lequel le monde prend d'autres valeurs.

La réalité de l'ailleurs représente une découverte surprenante pour le voyageur écrivain qui observe cette réalité d'un regard étranger, d'un regard qui témoigne de la curiosité pour la nouveauté. Le regard neuf du voyageur écrivain voit en la réalité de l'ailleurs un état sensationnel, un état qui ne peut pas être considéré dans les termes de l'ordinaire. Dans le contexte d'un tel rapport qui traduit le regard différent du sujet voyageur envers son environnement, c'est encore Roy qui explique l'expérience, en transmettant, en termes suivants, ses impressions lorsqu'elle se trouve dans la spatialité d'une ville étrangère: "j'eus le sentiment que c'était moi, l'étrangère de cœur avide, que la ville pendant ce moment s'était livrée plutôt qu'à ses habitants au regard usé. Et je restai sans savoir que faire de mon émerveillement" (2012, p. 286). La voyageuse canadienne raconte au lecteur comment se produit, quand il s'agit de la découverte d'un espace inconnu, une condition temporaire et exclusive concernant à la fois la perception curieuse du voyageur et la manifestation de l'ailleurs, contrairement au cas des "habitants au regard usé" qui restent en dehors d'une expérience de tel genre. II n'est pas difficile, à ce stade, de remarquer aussi un parallèle entre Kpomassie et Roy par rapport à leurs approches pour décrire la dissemblance entre la conception de l'espace des habitants et celle du voyageur. Comme l'expérience l'affirme, en rupture avec son titre d'habitant, le voyageur devient plus ouvert à ce qui se manifeste autour de lui et cède par conséquent à l'émerveillement qui est entrainé par les circonstances de sa découverte nouvelle. En ce cas, la réalité de l'espace obtient, à un moment donné, une autre forme qui se laisse éprouver pleinement et éventuellement par les sens du voyageur.

En parlant des valeurs, Victor Segalen atteste que son voyage prend "décidemment" pour lui “la valeur d'une expérience sincère: confrontation, sur le terrain, de l'imaginaire et du réel" (2018, p. 89). Ces mots nous renvoient à l'aspect imaginaire qui accompagne 
le côté réel du déplacement du voyageur écrivain lors duquel le réel et l'imaginaire se confrontent, autrement dit, le voyage devient le résultat du partage entre la réalité extérieure et l'imagination subjective. En voyage, l'espace prend un aspect inhabituel pour le voyageur qui sort de sa mode de vie sédentaire marquée par une indifférence envers son environnement. C'est la raison pour laquelle, Michel Onfray parle d'une "aptitude à la vision" que nécessite l'état du voyageur. Onfray, en employant le mot "nomade-artiste" en vue de décrire le voyageur écrivain que l'on tente de décrire dans ce travail, explique que "le nomade-artiste sait et voit en visionnaire, il comprend et saisit sans explications, par impulsion naturelle", en ajoutant que "dans ce cas, la réalité infuse par capillarité le voyageur qui appréhende" (2016, p. 65). Afin de souligner le caractère romanesque de l'expérience du voyageur écrivain, laquelle prend évidemment sa forme finale dans le récit de voyage, Onfray insiste sur l'origine de la motivation de partir ailleurs et résume que "[...] le désir de voyage se nourrit mieux de fantasmes littéraires ou poétiques que de propositions indigentes par trop de semblances avec une réalité sommaire" (2016, p. 23). À partir de ce point de vue qui est proposé par Onfray, se présente une conception du voyage qui peut être traduite par l'atténuation de la domination de la réalité sommaire vis-à-vis de la condition sensible de l'individu. Dans cette conception, la réalité objective s'avère inadéquate pour être la seule source de narration afin de réaliser un récit de voyage.

En se considérant en tant que nomade, le voyageur turc Nedim Gürsel associe son état de voyageur dans l'ailleurs à une solitude et cette solitude à "un autre être humain" qui s'est formé en lui sous forme d'un sentiment grandissant, d'un frissonnement indéniable. Gürsel, qui souvent crée un rapport entre l'expression poétique et ses voyages et qui prend la littérature de voyage pour une activité tournée vers la fiction, définit cet autre être humain comme un étranger absolu au début mais qui est devenu un véritable ami pour lui à partir du moment où il s'est mis à l'aimer et à s'habituer ainsi à son existence. Le voyageur turc explique que se trouver dans l'atmosphère étrangère d'un lieu lointain, parmi les gens que l'on connaît pas, est quelque chose qui a le pouvoir de renforcer le lien de cette amitié (2013, p. 20-21). En d'autres termes, en ce qui concerne sa solitude et ses sentiments extraordinaires, Gürsel évoque une valeur déterminante par rapport à son état de nomade étant intimement lié à la langue, aux mots qui se sont implantés dans sa mémoire au cours de sa vie et dont il fait usage, à son tour, pour transmettre au mieux possible ce qu'il expérimente dans un lieu étranger. Le récit de voyage devient donc l'histoire de cet autre être humain traduit par les mots choisis du voyageur écrivain tentant de produire un langage subjectif. 
La rhétorique du voyageur écrivain est inséparable de sa subjectivité qui se révèle à travers l'expression des sensations, des rêves, des changements psychiques et physiques apparaissant sous l'inévitable influence de l'ailleurs. Le récit de voyage fournit, en ce sens, à côté de la réalité extérieure, cet aspect non objectif que l'on découvre dans les mots du voyageur écrivain qui reflètent un aspect intime et personnel relativement à cette réalité extérieure découverte. L'essentiel du récit de voyage est ainsi dû à ce rapport qui ne se développe qu'entre un voyageur observateur et un espace étranger.

Tel qu'il est possible de le constater, Onfray attribue une valeur singulière au récit de voyage écrit par l'artiste-nomade et dans lequel on trouve un monde qui est reproduit à partir de ce que l'auteur a perçu, a senti et a imaginé. Il s'agit ici d'une image tout à fait individuelle qui porte essentiellement des traits romanesques propres au moment réellement vécu dans la spatialité de l'ailleurs. De cette manière, un espace quelconque est en mesure de prendre, sous la plume du voyageur écrivain, une image extraordinaire, et c'est en effet cette image qui amène ce dernier à s'approprier un rôle de narrateur exceptionnel. Or, dans le récit de voyage, les mots, les expressions et les descriptions du voyageur écrivain présentent au lecteur une perspective d'entendre le monde qui n'est pas semblable à aucune autre. Il s'agit là d'une œuvre littéraire qui est une preuve singulière dans laquelle prend forme une conception de l'espace qui relève, d'une part, de la particularité des sens et de l'imagination du voyageur, d'autre part, de la condition particulière de l'ailleurs. Ces deux aspects conceptuels sont, certes, des aspects relatifs, étant donné que l'ailleurs prend son caractère romanesque d'après la vision du voyageur écrivain qui est nourrie par l'imaginaire et par le sensationnel, et, par ailleurs, il faut le rappeler, c'est la condition de l'ailleurs qui provoque les sens et l'imagination du voyageur écrivain.

La réalité change, pour ainsi dire, de sens dans le cas du voyageur écrivain qui se trouve dans l'ailleurs, et cet aspect changé de la réalité, en raison de sa particularité qui s'écarte de l'ordinaire et qui est fortement marquée de sentiments très divers, se figure sous une forme étrange, c'est-à-dire, il présente les traits d'une réalité surprenante et excentrique. De même, au moment de son déplacement loin de son chez-soi, la personnalité du voyageur écrivain se révèle dans un nouvel état qui fait preuve d'une autre manière d'être dans l'espace. Cet état éventuel trouve, par conséquent, son image accomplie dans le récit de voyage à l'intérieur duquel les mots, les expressions et les descriptions se réfèrent à la traduction d'une réalité exclusivement et passagèrement découverte par son auteur qui, par rapport à ce qu'il a rencontré et a éprouvé pendant 
son séjour dans la spatialité imprévisible du non-chez-soi, montre la volonté de transmettre son expérience personnelle à travers un langage qu'il constitue de façon à faire penser à une rhétorique intime. Une entreprise pareille provenant de la part du voyageur écrivain dénote sans doute un acte de témoignage qui correspond à une situation singulière marquée d'une part par la réalité extérieure et objective, d'autre part, par une subjectivité imaginaire et sensible, une situation que l'on peut décrire comme une manière d'être romanesque dans l'espace.

\section{Conclusion}

Transmettre l'expérience de voyage en mots se rapporte à un engagement qui est le résultat de la découverte d'une autre manière d'être. Cela étant, écrire le voyage peut être considéré comme un projet romanesque par lequel le voyageur écrivain est censé transmettre les éléments remarquables d'un temps ayant vécu par lui, sous des conditions extraordinaires. En voyage, en effet, comme l'espace se manifeste sous une différente image, le voyageur écrivain, dont la perception et l'imagination se soumettent à éprouver des circonstances inhabituelles, devient Autre. De là, quand le voyageur devient écrivain, il entreprend de raconter, par l'intermédiaire de son récit individuel, l'histoire de cet Autre qui représente un sujet étranger ayant perçu l'espace d'un regard observateur et affectif et cela pour un certain temps. "Le voyage les transformait, ils décrivaient leur métamorphose, cet autre qui naissait en eux" (2017, p. 13), résume Laurent Maréchaux pour fournir une explication à cette situation exceptionnelle qui définit le processus par lequel le voyageur effectue son acte de déplacement. Puisque le récit de voyage, en tant que création littéraire comprenant des éléments qui sortent de l'ordinaire, de I'habituel, de l'attendu et du commun, devient la preuve finale et la plus concrète de cette métamorphose qui définit l'expérience du voyageur écrivain, c'est uniquement sous la forme d'un ouvrage romanesque que le lecteur acquiert la possibilité de savoir sur ce déplacement.

Généré par une décision aléatoire de partir ailleurs, le déplacement du voyageur écrivain donne lieu à une manière d'être romanesque dans l'espace, et le récit de voyage, qui contient l'image d'un monde extérieur, concret et objectif étant associé avec un monde personnel, affectif, changeant et imprévu, devient la preuve de cette expérience tout à fait personnelle. Dans son œuvre, en qualité de narrateur qui s'est engagé à transmettre au lecteur une certaine impression du monde d'après les circonstances de l'ailleurs, le voyageur écrivain raconte donc les détails d'une différente condition 
d'espace, laquelle ne pourrait pas être vécue dans les limites déterminées du chez-soi. Dans chaque récit de voyage, l'espace est ainsi réinterprété et reproduit relativement à ce qui fut expérimenté par le voyageur écrivain à titre de sujet étranger observant son environnement inconnu d'un regard sensible et imaginaire.

Réalisés au cours des vingtième et vingt-et-unième siècles et abordant le thème de voyage, les ouvrages sélectionnés comme références pour l'élaboration de ce présent travail, nous a mené à reconnaître un aspect romanesque en ce qui concerne la conception de l'espace qui est présentée dans les récits de voyage contemporains. Cet aspect, caractérisé par la contexture du réel et de l'extraordinaire quant à percevoir l'espace d'une perspective individuelle et nouvelle, est affirmé dans chaque source citée, quoi que ce soit en termes plus ou moins différents, en nous permettant de le considérer comme un phénomène de littérature de voyage. Dans cet article, les témoignages et les remarques rapportés et comparés l'ont attesté que le voyageur écrivain représente une figure littéraire qui fait penser à la notion "romanesque" en raison de sa manière de transmettre, de raconter, dans son récit de voyage, ce qu'il a vécu relativement à la réalité qu'il a expérimentée et a ressentie dans un espace situé loin de sa sphère habituelle. En considération de ces témoignages et remarques venant des auteurs représentant les divers périodes des deux derniers siècles, il est, après tout, possible d'observer d'une manière explicite une concordance entre le titre de « voyageur écrivain » et celui de "voyageur romanesque" qui indiquent en même temps une association conceptuelle et un rapport consécutif lorsqu'il s'agit de transmettre, dans le cadre de la littérature de voyage, l'expérience de déplacement acquise dans la spatialité de l'ailleurs.

Évaluation : Évaluation anonyme par des pairs extérieurs.

Conflit d'intérêts : L'auteur n'a aucun conflit d'intérêts à déclarer.

Subvention : L'auteur n'a reçu aucun soutien financier pour ce travail.

Peer-review: Externally peer-reviewed.

Conflict of Interest: The author has no conflict of interest to declare.

Grant Support: The author declared that this study has received no financial support.

\section{Notes}

1. Voir Portail lexical du Centre National de Ressources Textuelles et Lexicales, "Romanesque", https://www.cnrtl. $\mathrm{fr} /$ definition/romanesque (consulté le 10 juin 2020). 


\section{Bibliographie}

Batur, E. (2011). Yolcu. İstanbul: Kırmızı Yayınları.

Bouvier, N. (2001). L'usage du monde. Paris: Éditions Payot\&Rivage.

Gürsel, N. (2013). Bir avuç dünya. İstanbul: Doğan Kitap.

Kpomassie, T.-M. (1981). L'Africain du Groenland. Paris: Flammarion.

Maréchaux, L. (2017). Écrivains voyageurs: Ces vagabonds qui disent le monde. Barselona: Éditions Arthaud.

Onfray, M. (2016). Théorie du voyage: poétique de la géographie. Sant Andreu de la Barca: Librairie Générale Française.

Richemont, B. de (2015). Manifeste vagabond. Paris: Presses de la Rennaissance.

Roy, G. (2012). La Détresse et l'Enchantement. Cap-Saint-Ignace: Boréal.

Segalen, V. (2018). Essai sur l'exotisme. Paris: Librairie Générale Française.

Tesson, S. (2017). Éloge de l'énergie vagabonde. Malesherbes: Éditions des Équateurs. 
